\title{
195. Ausgleich von Stirnbeindefekten mit autologem Knorpel
}

\author{
F. Nagel \\ Krankenhaus Siloah, Wilferdinger Straße 67, D-7530 Pforzheim
}

\section{Augmentation of Frontal Bone Defects by Autogenous Cartilage Implant}

Summary. The pre-fabrication of cartilage and connective tissue from ribs is ideal for the reconstruction of the forehead. Plastic moulds shaped around a wax model corresponding to the defect are implanted under the abdominal skin and left there for 6 months. The penetrating connective tissue produces a solid, strong form. Complications did not occur and resorption has not been observed.

Key words: Cartilage implant - Forehead.

Zusammenfassung. Der vorgefertigte Knorpel-Bindegewebs-Span ist für den Stirnaufbau ideal. Er wird gebildet, indem Chips aus Rippenknorpel gewonnen werden. Diese Chips werden in Kunststoffschalen gefüllt, die um ein zuvor angefertigtes Wachsmodell angefertigt wurden. Das Wachsmodell entspricht dem Defekt. Die Kunststoffschalen werden für sechs Monate unter die Bauchhaut implantiert. Durch einwachsende Bindegewebssepten entsteht ein solider vitaler Span. Komplikationen wurden bei diesem Vorgehen nicht beobachtet. Resorptionserscheinungen traten nicht auf.

Schliisselwörter: Knorpelspanimplantation in Stirnbeindefekt.

\section{Hydroxylapatitkeramik als Knochenersatzmaterial - experimentelle und klinische Ergebnisse}

\author{
J. F. Osborn und G. Pfeifer
}

Nordwestdeutsche Kieferklinik der Universität Hamburg, Martinistraße 52, D-2000 Hamburg 20

\section{Hydroxyapatite Ceramic for Bone Replacement - Experimental and Clinical Results}

Summary. During the last 2 years hydroxyapatite ceramic has been implanted into 18 patients. The frontal region of atrophic mandibulas was augmented with interpositional application of porous hydroxyapatite ceramic in block form. Cysts of the jaw and defects resulting from dental implant failure were filled with hydroxyapatite ceramic granules. Alveolar collapse was prevented by implanting hydroxyapatite granules into the sockets after extraction of teeth. Histologic evaluation of bone biopsies demonstrates that hydroxyapatite ceramic is integrated into human jaw bone physiologically.

Key words: Implantology - Bone replacement - Hydroxyapatite ceramic.

Zusammenfassumg. In einem Zeitraum von mehr als 2 Jahren wurde Hydroxylapatitkeramik bei 18 Patienten implantiert - in Form poröser Blöcke zur Erhöhung der Unterkieferfront in Sandwich-Technik oder als Granulat zur Füllung von Cysten und von Defekten nach dem Verlust von Dental-Implantaten. In leere Alveolen eingebracht verhindert Hydroxylapatit-Granulat den alveolären Kollaps. Histologische Auswertungen zeigen, daß Hydroxylapatitkeramik die gleiche physiologische Integration in menschliches Knochengewebe aufweist, die wir seit 1978 in zahlreichen Tierversuchen gefunden haben.

Schliusselwörter: Implantologie - Knochenersatz - Hydroxylapatitkeramik. 